\title{
Lecturing on Silicon Sensors using the Educational Alibava System
}

\author{
J. Bernabeu ${ }^{a}$, G. L. Casse $^{b}$, C. García ${ }^{a}$, C. Jumilla ${ }^{d}$, C. Lacasta ${ }^{* a}$, M. Lozano $^{c}$, \\ S. Martí ${ }^{a}$, G. Pellegrini ${ }^{c}$, J. Rodriguez $^{d}$, M. Ullán $^{c}$ \\ ${ }^{a}$ Instituto de Física Corpuscular, IFIC/CSIC-UVEG, Valencia, Spain \\ ${ }^{b}$ Instituto de Microelectrónica de Barcelona, IMB-CNM/CSIC, Barcelona, Spain \\ ${ }^{d}$ University of Liverpool, Liverpool, United Kingdom. \\ ${ }^{d}$ Alibava Systems, Barcelona, Spain \\ E-mail: infodalibavasystems.com
}

\begin{abstract}
The Educational ALIBAVA System (EASy) is a compact and portable system for lecturing sensor instrumentation at university teaching laboratories. EASy tests and reads out a silicon micro-strip sensor. The front-end electronics is based on a low noise ASIC with 128 input channels. Semiconductor devices are widely used as radiation sensors in many scientific and industrial applications. They are of uttermost importance in the High Energy Physics experiments as tracking devices and extensively used in Nuclear Physics for spectroscopy. Moreover, silicon sensors are extensively used in medical physics imaging and inland security, where their low cost, miniaturisation, packaging and integration of electronics represent a clear advantage. A book of exercises with EASy (including theory explanations and hands-on activities) helps physics and engineering students to be educated on the first principles of the semiconductor sensors, their operation in real systems, as well as the associated electronics.
\end{abstract}

The European Physical Society Conference on High Energy Physics

22-29 July 2015

Vienna, Austria

${ }^{*}$ Speaker. 


\section{Introduction}

The ALIBAVA system [1] is the first compact and portable system for the characterisation of silicon microstrip radiation detectors. It is a plug \& play system that can be controlled from a graphical user interface that runs in the most common computer operative systems: Windows, Linux and Mac OSX. The main components of the system are a mother board containing a FPGA that manages all the operation modes, the communication with the computer and configures the readout chips. There is a second board, the daughter board, that houses the sensors and the readout chips.

The ALIBAVA system was developed by the University of Liverpool, IMB-CNM ${ }^{1}$ in Barcelona and IFIC $^{2}$ in Valencia with the idea of producing a standardised, compact and easy to use setup to characterise silicon micro strip sensors. The development was supported by the CERN-RD50 [2] collaboration to find the technology for the silicon trackers and vertex detectors of the experiments at the High Luminosity Upgrade of the LHC [3]. The system was very soon widely used in the community and the ALIBAVA Systems spin-off company was created to improve and distribute the system. Today, the system is used over 30 particle physics institutes in Asia, Europe and America.

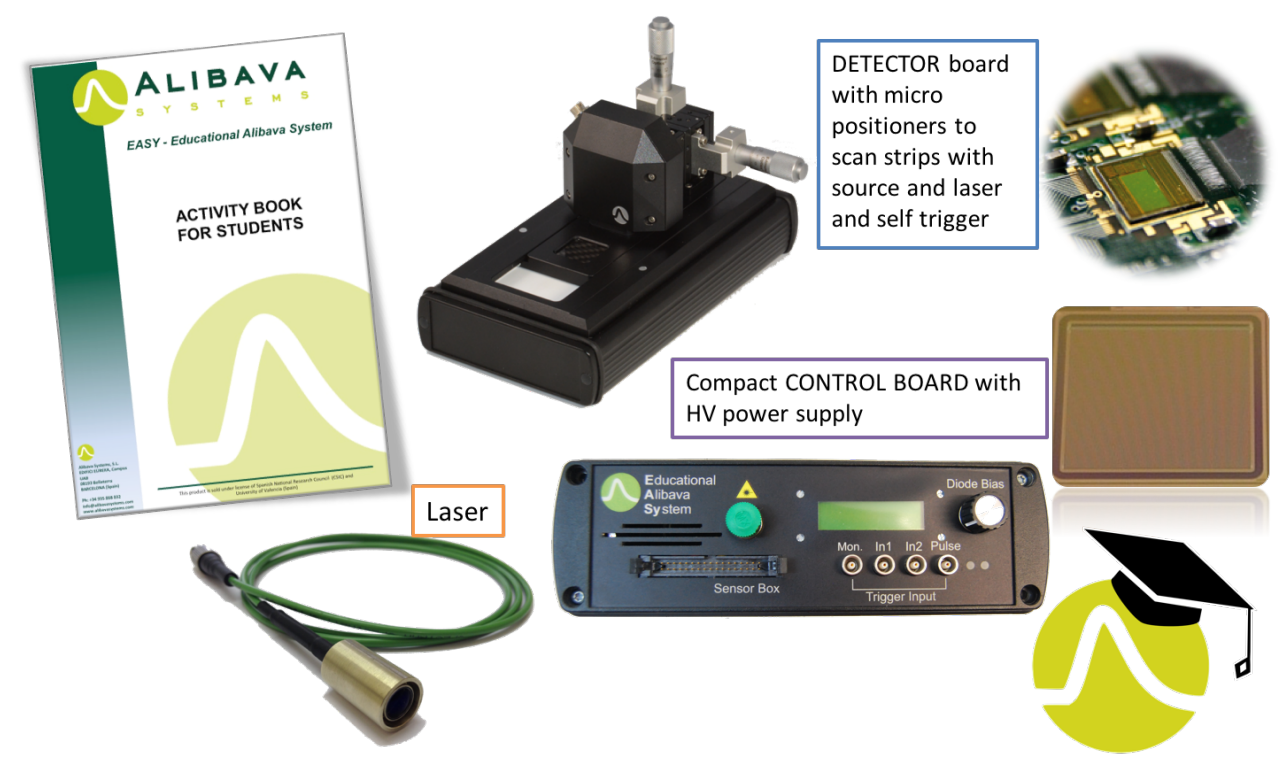

Figure 1: The components of the EASy system.

A natural evolution of the system was a specialisation on education and training which brought the Educational Alibava System, EASy, into the game. Fig. 1 shows the various componentes of the system.

The EASy features were developed with the idea of fully exploiting the capabilities of the system while simplifying its setup and helping to understand the basic principles and operation of the semiconductor devices:

\footnotetext{
${ }^{1}$ Instituto de Microelectrónica de Barcelona, CSIC

${ }^{2}$ Instituto de Física Corpuscular, CSIC-UVEG
} 
1. The system incorporates a HV unit to bias the sensor.

2. The system includes an infrared laser to point to individual channels with a micro-positioner.

3. Encapsulating box that protects against accidents and misuse.

4. Acquisition software and data analysis macros adapted to the experiments manual.

5. Experiments manual with basic experiments with a laser and radioactive sources.

In addition, the system has self-triggering capabilities, plus a silicon diode, which may be used for triggering the acquisition. Thus, there is no need of an external trigger generator.

EASy includes an infrared laser mounted on a focusing system to point individual channels, study charge sharing between channels and study the laser penetration in the semiconductor sensors. For these studies, EASy generates its own periodic trigger. The system comes with a step by step guide that sets the basic theory concepts and explains how to operate with it, with a description of the different acquisition modes. The guide also contains a proposal of basic exercises for the students like: setting the operation voltage of the sensors, finding the pedestals and the noise, the synchronisation with the trigger, charge collection and energy deposition, depletion voltage of the sensor, charge sharing between neighbour strips, the spatial resolution of the sensor and spectroscopy with different radioactive source.

\section{The control unit.}

The control unit is the equivalent to the mother board in the Alibava System. It is the only component, together with the computer that needs to be plugged in to the wall socket. The control unit has the brain that controls the whole system according to the commands received from the DAQ software running on the computer.

It provides the high voltage for depleting the sensor and measures the sensor current to help making IV-curves to characterise the sensor. The board also generates the operation voltages for the readout chips on the sensor unit and sends them the configuration commands as well as trigger, clock and control signals. This is sent with a flat cable connected in (2) of Fig. 2.

The control unit has, also, a $980 \mathrm{~nm}$ laser source. The $5 \mathrm{~ns}$ wide light pulses are sent to the sensor unit via an optical fibre, (1) in Fig. 2, into the focusing system in the daughter board.

The control unit is connected to the
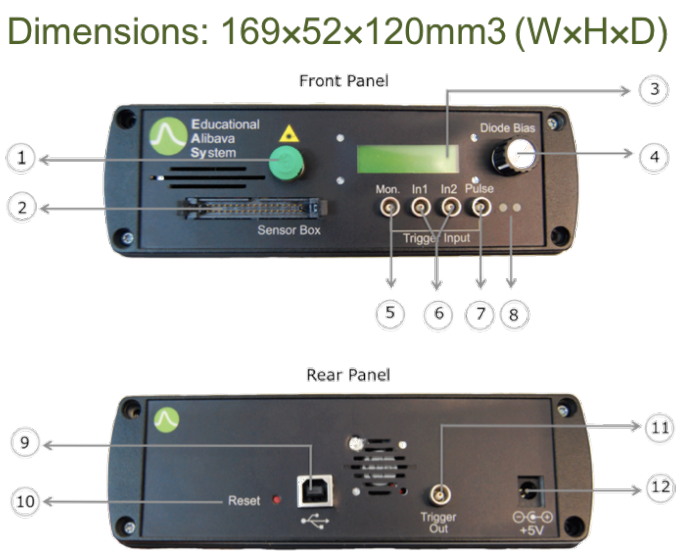

Figure 2: The control unit computer via USB and it is through that bus that data are commands are transmitted from one side to the other. 


\section{The sensor unit.}

The sensor unit contains the sensor, the front-end electronics and a diode to provide a trigger for the acquisition. The sensor is a $2 \times 2 \mathrm{~cm}^{2}$ silicon strip detector with a $150 \mu \mathrm{m}$ strip pitch. There is a silicon diode, matching the size of the sensor, at the back side of the board as can be seen in Fig. 3.

The read out ASIC used in this board is the Beetle chip [4]. It is a 128 channel chip providing analogue information on the amplitude of the signals connected at the input. It has, also, some self-triggering capabilities. Fig. 3.a shows the two sides of the electric board containing the sensor, diode and front-end electronics.

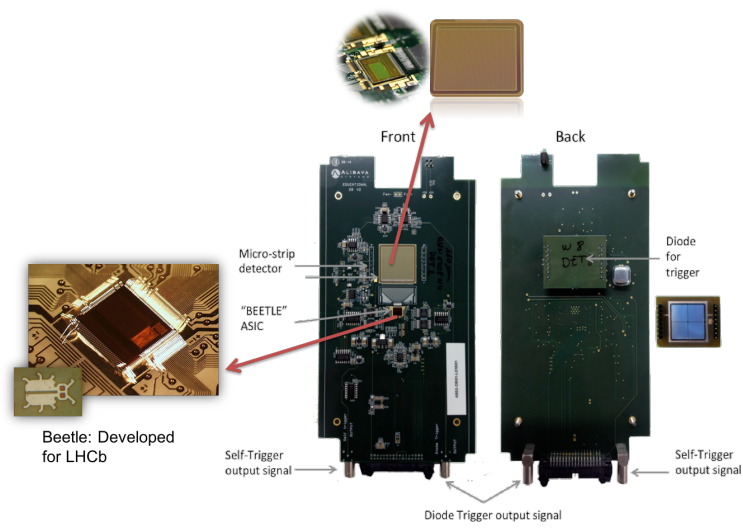

(a)

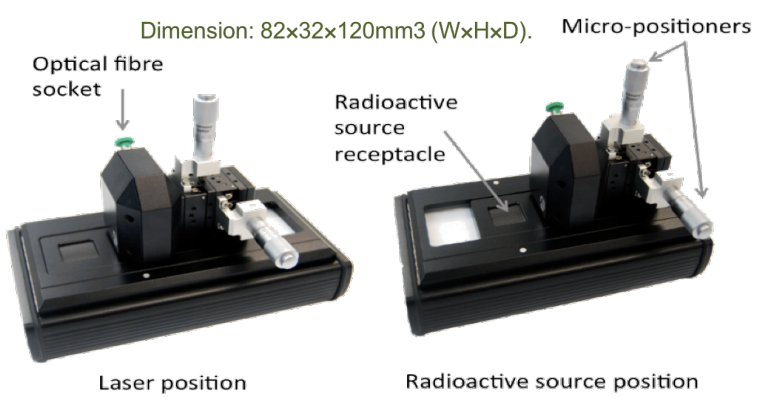

(b)

Figure 3: (a) Boths sides of the sensor unit are shown with the main components blown up to show the details. (b) Detail of the sensor unit box showing the two positions for laser and radioactive source runs. The micropositioners can also be seen.

The electric board is housed by a light-tight box with inputs for the electrical signals coming from the control units as well as for the fibres transmitting the laser pulse generated at the control unit. This can be appreciated in Fig. 3.b.

The box has a sliding cover with two positions. The first is an opening covered with a carbon fibre slab which is the receptacle of a radioactive source. This opening is on a structure that can be moved across the sensor strips, with the help of micro-positioners, so that one can study charge sharing effects with the source. The second position has a focusing system for the laser pulse coming from the control unit. The beam spot can be focused on the sensor surface achieving a beam spot of a few micrometers. The focusing system uses a second micro-positioner.

\section{The DAQ snd analysis software}

The system includes a specific data acquisition software and data analysis tools to demonstrate and visualise the operating principles of the silicon strip detector. The main window of the graphical user interface provides the means to configure the readout chip and the system as a whole. It is able to manage the different calibrations procedures for the front-end and the self-triggering discriminator. It has, also, a number of tabs showing different histograms that allow to monitor 
important aspects the data acquisition process like pedestals, noise, signal spectrum and, also, an event display that helps understanding the signal formation in the silicon sensor.

The software allows to save the data acquired in files for further analysis. The system provides, together with the DAQ software, a generic data analysis set of tools written for the most common data handling software: Matlab [5], Octave [6] and ROOT [7].

\section{Activity book for Students}

The kit incorporates the user manual and a specific exercises book [8] ideal for introducing the student on the high energy physics/particle physics experiments. The Student's activity book gives a general introduction to the most basic concepts of the operation of the silicon detectors like signal formation, spatial resolution, sources of noise and the different trigger modes.

It proposes up to 10 experiments with exercises as well as small pieces of software to analyse the data and produce some figures out of that. The exercises cover important aspects like how to produce the characteristic IV curve of the sensor, introduce the concepts of pedestals, noise and coherent noise, cluster finding algorithms, charge collection efficiency, energy deposition, spatial resolution, laser penetration in silicon and spectroscopy.

Fig. 4 show, with some more detail, four of the proposed exercises, their goals an the expected results from the data analysis.

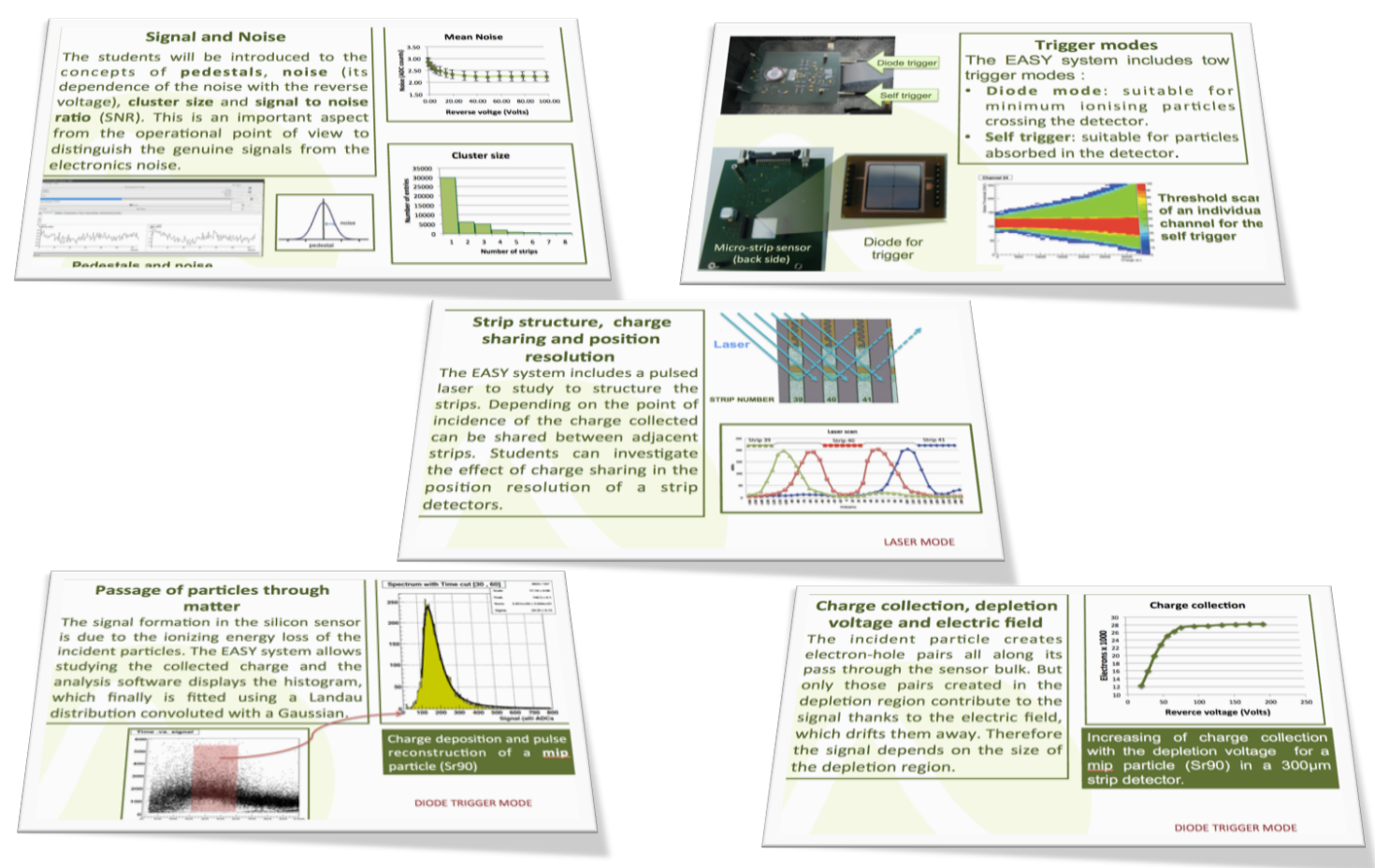

Figure 4: A more detailed description of four of the proposed exercises in the student's activity book. 


\section{Conclusions}

The system is a perfect tool to learn about the signal formation in semiconductor sensors and its processing. It is an ideal tool for Physics and Engineering students following courses on radiation detection instrumentations. It provides a number of tools to understand and analyse the data coming from the detector for the physics students and, also, provides means of playing with more internal aspects of the system like ASIC control, etc. for the engineering students.

It is a very compact system, see Fig. 5, suitable for experiments in the laboratory, and for outside experiments in schools, tutorials, etc. thanks to its portability.
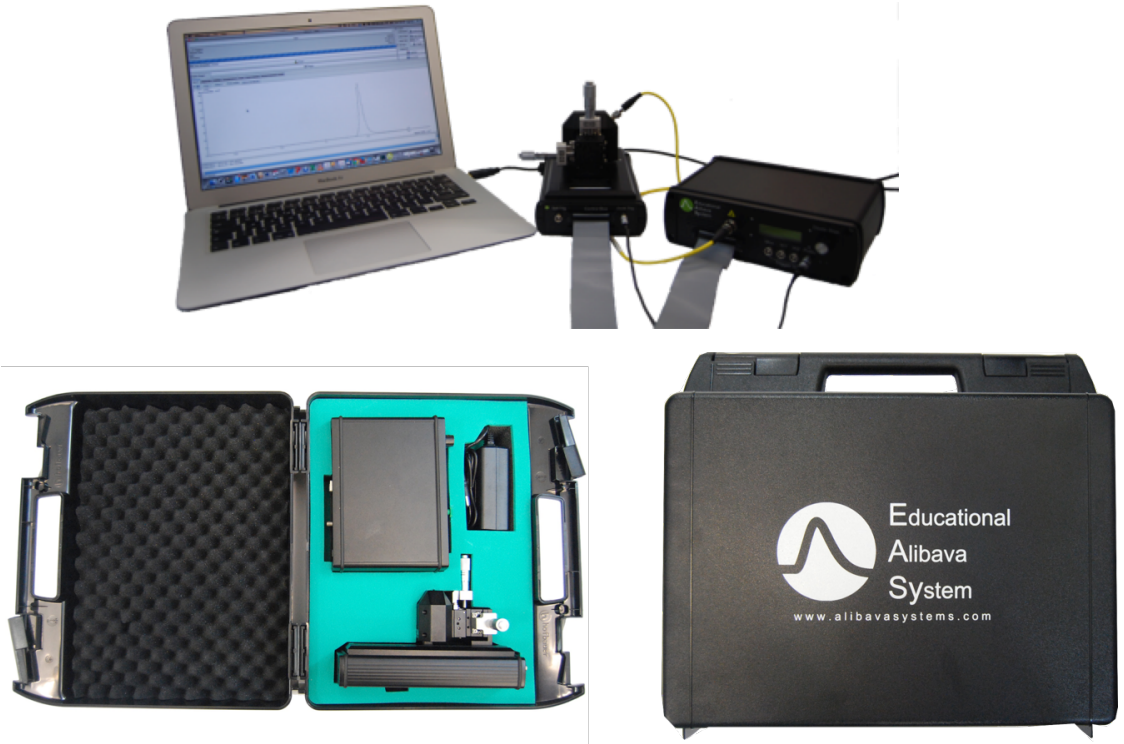

Figure 5: The ALibava Educational System in a box.

\section{References}

[1] R. Marco. A portable readout system for microstrip silicon sensors. IEEE Trans.Nucl.Sci. Vol. 56, pp.1642-1649, 2009 doi:10.1109/TNS.2009.2017261.

[2] RD50 - Radiation hard semiconductor devices for very high luminosity colliders. http://rd50.web.cern.ch

[3] The High Luminosity Large Hadron Collider. Ed. O. Brüning and L. Rossi. World Scientific. ISBN: 978-981-4675-46-8

[4] S. Loechner, Development, Optimisation and Characterisation of a Radiation Hard Mixed-Signal Readout Chip for LHCb, Phd. thesis, Max-Planck Institute for Nuclear Physics Heidelberg, 2006.

[5] MATLAB. Natick, Massachusetts: The MathWorks Inc., 2010.

[6] John W. Eaton, David Bateman, and Søren Hauberg (2009). GNU Octave version 3.0.1 manual: a high-level interactive language for numerical computations. CreateSpace Independent Publishing Platform. ISBN 1441413006, URL http://www.gnu.org/software/octave/doc/interpreter/

[7] R. Brun, and F. Rademakers, ROOT - An Object Oriented Data Analysis Framework. Nucl. Inst. Meth. in Phys. Res. A 389 (1997) 81-86.

[8] Downloadable from the Alibava Systems web page: http://www.alibavasystems.com 